\title{
DETERMINACIÓN SEXUAL: ¿CÓMO ESTRUCTURA LA BIOMEDICINA CONTEMPORÁNEA SU DISCURSO SOBRE LA GÉNESIS DE LA DIFERENCIA? UN ESTUDIO CON FOCO EN ARGENTINA
}

\author{
SEX DETERMINATION: HOW DOES CONTEMPORARY \\ BIOMEDICINE STRUCTURE ITS DISCOURSE ON \\ THE GENESIS OF DIFFERENCE? A STUDY FOCUSED \\ ON ARGENTINA
}

Authors / Autoras:

Luciana Hadid

Universidad de Buenos Aires

Buenos Aires, Argentina

luhadid@gmail.com

https://orcid.org/0000-0002-7446-871X

\section{Marcela Belardo}

Universidad Nacional de José C. Paz

Universidad de Buenos Aires

Buenos Aires, Argentina

marcelabelardo@yahoo.com.ar

https://orcid.org/0000-0001-9032-3919

Submitted / Recibido: 16/03/2020

Accepted / Aceptado: 02/11/2020

To cite this article / Para citar este artículo: Hadid, L. y Belardo, M. (2021).

Determinación sexual: ¿cómo estructura la biomedicina contemporánea su discurso sobre la génesis de la diferencia? Un estudio con foco en Argentina. Feminismo/s, 38, 331-357. https://doi.org/10.14198/ fem.2021.38.13

Licence / Licencia:

This work is licensed under a Creative Commons Attribution 4.0 International.

\section{(c) (1)}

(c) Luciana Hadid y Marcela Belardo

\author{
Luciana HADID y \\ Marcela BELARDO
}

\section{Resumen}

El discurso biomédico contemporáneo sostiene que el elemento determinante del sexo humano es el camino de desarrollo tomado por las gónadas al comienzo de la vida. A través de un proceso conocido como determinación sexual, esas estructuras dejan de ser órganos sexualmente indiferenciados para transformarse en ovarios o testículos, en un enfoque binario. Eso define el resto del desarrollo genital. El objetivo de este artículo es identificar quiénes elaboran el discurso biomédico sobre determinación sexual entre 1990 y 2015 , y qué materiales producen. Este análisis está enmarcado en la propuesta teórica de Ludwik Fleck, a partir del concepto de colectivo de pensamiento. La metodología es cualitativa, con fuentes primarias y secundarias. La determinación sexual es enunciada como un proceso estrictamente natural que no constituye un objeto epistemológico. Este discurso perpetúa 
condiciones opresivas y normalizadoras que resultan centrales en la estructuración de la verdad biomédica sobre el cuerpo normal.

Palabras clave: determinación sexual; sociología de la ciencia; biología del desarrollo; género; diferencia sexual.

\begin{abstract}
Contemporary biomedical discourse holds that the determining element of human sex is the path of development taken by the gonads at the beginning of life. Through a process known as sex determination, these structures cease to be sexually undifferentiated organs to become ovaries or testicles, in a binary approach. That defines the rest of the genital development. The objective of this article is to identify who elaborate the biomedical discourse on sexual determination between 1990 and 2015, and what materials they produce. This analysis is framed in the theoretical proposal of Ludwik Fleck, based on the concept of thought collective. The methodology is qualitative, with primary and secondary sources. Sex determination is enunciated as a strictly natural process that does not constitute an epistemological object. This discourse perpetuates oppressive and normalizing conditions that are central to the structuring of biomedical truth about the normal body.
\end{abstract}

Keywords: Sex determination; Sociology of science; Developmental biology; Gender; Sexual difference.

\title{
INTRODUCCIÓN
}

La biomedicina contemporánea explica el desarrollo genital prenatal centrándose en el desarrollo gonadal y sostiene que la determinación del sexo de una persona ocurre, en primer término, en las gónadas embrionarias. Desde una perspectiva binaria, consiste en la transformación de las gónadas embrionarias indiferenciadas de las primeras semanas de gestación en testículos o en ovarios. Este proceso es conocido como determinación sexual (Gilbert, 2010). A partir de esta determinación se produce, de acuerdo con el discurso biomédico actual, la diferenciación del sexo del resto del cuerpo del producto de la gestación. Aparecen entonces dos categorías definidas en el desarrollo prenatal: la determinación sexual -definición del sexo de las gónadas embrionarias- y, a partir de esta, la diferenciación sexual -resto de los órganos del embrión- (Gilbert, 2010). La diferencia sexual es presentada

Feminismo/s 38, July 2021, 331-357 
como un hecho natural y su génesis se ubica durante el desarrollo prenatal, en particular en el proceso de determinación sexual. En este relato, el sexo se estructura antes de nacer a través de una definición binaria: tomar el camino de uno de los dos sexos posibles.

La biomedicina suele analizar sus hechos científicos desde una perspectiva ahistórica, tomando como eje el descubrimiento de una verdad natural por parte de un observador objetivo (Fleck, 1986; Foucault, 1998, 2003; Serrano, 2007). Esta mirada contiene en sí misma la falta de reflexión crítica sobre su epistemología (Fleck, 1986). Entre las características específicas de la determinación sexual como objeto de estudio destaca el hecho de que está atravesada por un particular interjuego entre lo universal y lo íntimo, lo individual y lo colectivo, lo normal y lo patológico, la dicotomía sexual y las diversidades, las moléculas y las identidades. Esta singularidad hace de la determinación sexual un objeto privilegiado para estudiar cómo y en qué medida la manera en que la biomedicina construye sus objetos tiene sesgos que constituyen límites. Y también, especialmente, consecuencias.

El discurso biomédico -como cualquier otro- no relata objetivamente un hecho dado, sino que es un desarrollo en el que determinados actores sociales crean los patrones de la vida social a través de sus concepciones y sus acciones (David y Sutton, 2004). Constituye parte de la realidad histórica de esos actores y por lo tanto resulta central en la definición de sus prácticas. En esta línea, no es suficiente con que la reflexión en torno al discurso biomédico se haga sobre las técnicas y proposiciones de la biomedicina, sino que debe incluir el análisis de los patrones de racionalidad y aplicación que se encarnan en esas técnicas y proposiciones, así como en otras formaciones discursivas (Foucault, 1979) que componen el tejido social (Canguilhem, 1971; Safatle, 2011). Este discurso es entonces una construcción social. En tanto social, esta construcción es colectiva y no puede explicarse a través de la suma de aportes individuales: implica el desarrollo de un colectivo de pensamiento que lo elabora, de acuerdo con un estilo de pensamiento determinado (Fleck, 1986).

En relación con el recorte temporal seleccionado, se destaca que en 1990 se publicó en la revista Nature, de referencia en biomedicina, la descripción de un gen humano llamado SRY (Sex Determining Region Y) en el cromosoma Y (Sinclair et al., 1990). El hallazgo se presentó como la condición 
necesaria y suficiente para que un embrión humano desarrolle testículos. Se postuló que su carencia redunda, a su vez, en la formación de ovarios. Esta especificación de la región determinante del sexo marcó un hito en el conocimiento de la determinación sexual humana y tuvo gran repercusión en la comunidad científica (John Hopkins University, 2017; Mc Laren, 1990; Nagai, 1996; Nordqvist, 1995; Ronfani y Bianchi, 2004).

Este trabajo se centra en identificar quiénes elaboran este discurso y qué materiales producen, y se organiza en tres secciones. En la primera sección del artículo se detalla la metodología utilizada. En la segunda sección se describe el centro del colectivo de pensamiento biomédico que se organiza en torno a la determinación sexual, en clave de analizar cómo construye su discurso. En la tercera sección se caracterizan las principales producciones escritas de este colectivo.

\section{CONSIDERACIONES METODOLÓGICAS}

Se seleccionó para esta investigación un diseño exploratorio y descriptivo. La estrategia metodológica fue cualitativa y consideró el discurso biomédico sobre determinación sexual como construcción colectiva. Se trabajó sobre la base de fuentes orales y textuales. La recolección de datos se efectuó por medio de una articulación que incluyó fuentes primarias a través de entrevistas a especialistas y fuentes secundarias a partir de la revisión de dos tipos de documentos: una selección de artículos publicados en revistas biomédicas sobre la temática entre 1990 y 2015, y los 3 manuales de estudio más recomendados para su abordaje en las escuelas públicas de medicina de la Argentina. El corpus empírico -en particular la selección de artículos- se fue redefiniendo a medida que avanzaba el trabajo de campo. En términos de interacción con los datos, se elaboró en un primer momento una selección inicial de manuales y artículos. Luego se elaboró la guía de entrevistas y se las llevó a cabo. A partir de las entrevistas se efectuó la selección final de artículos y el análisis de datos. Este artículo concentra un recorte de una investigación más amplia. 


\section{Las entrevistas}

Se efectuaron seis entrevistas semi-estructuradas. Para ello, se seleccionaron dos personas expertas en la temática (que trabajan en diferentes centros y son quienes más se destacan en investigación sobre desarrollo genital prenatal humano en Argentina, con inserción en el colectivo internacional) y cuatro especialistas que trabajan en áreas asociadas. Se incluyeron trabajadores de las áreas de la docencia, la investigación y la asistencia, y dentro de esta última a personas experimentadas en diversas disciplinas clínicas y quirúrgicas (cirugía general y ginecológica pediátrica, endocrinología pediátrica, pediatría, genética médica, investigación básica y clínica, docencia de grado y posgrado). Se evita caracterizar con mayor detalle para preservar el anonimato.

La guía de entrevistas se estructuró en 3 ejes: conocimiento sobre determinación sexual, estructura del colectivo de pensamiento y su producción, y transmisión de conocimiento sobre determinación sexual en las carreras públicas de medicina de la Argentina. A partir de las entrevistas se terminó de localizar el discurso sobre determinación sexual en cuanto a la estructura del colectivo de pensamiento y su producción.

\section{Los artículos}

Se seleccionó una serie de artículos indexados en la base de datos especializada más utilizada por el colectivo biomédico a nivel mundial: MEDLINE (Fernández-Altuna et al., 2016).

En primer lugar, la selección de artículos científicos se efectuó en MEDLINE a través del motor de búsqueda PubMed, utilizando el término $\mathrm{MeSH}^{1}$ sex determination processes. MeSH define este término como «los mecanismos por los cuales el sexo de las gónadas de un individuo es fijado» (traducción propia). Se utilizó el término seleccionado como major topic (es decir, dentro de los temas principales del artículo). Como filtros de la búsqueda se introdujeron la fecha de publicación entre el 1 de enero de 1990 y el 31 de diciembre de 2015, la especie humana, y el idioma: inglés, castellano y portugués. A pesar de haber filtrado por especie y tema, se obtuvieron

1. Glosario de PubMed.

Feminismo/s 38, July 2021, 331-357 
numerosos artículos que no cumplían con estos requisitos. Mediante la lectura de título y resumen se descartó otro grupo: quedaron 264 artículos.

Se efectuó una búsqueda adicional porque el término MeSH sex determination processes fue introducido en 2011 y los artículos anteriores fueron indexados utilizando este término retrospectivamente hasta 1998. Con el término MeSH sex differentiation como major topic (se utiliza como relativo al desarrollo sexual prenatal en general) para el período 1990-1997, después de la revisión inicial de título y resumen, quedaron 89 artículos adicionales.

De estas búsquedas no surgió el artículo con el que el grupo de Sinclair comunicó la descripción del gen SRY humano en la revista Nature (Sinclair et al., 1990). Como constituyó el punto de inflexión en el conocimiento biomédico sobre determinación sexual que marca el inicio del recorte temporal seleccionado para este trabajo, ese artículo fue incluido.

Fue necesaria la lectura de los textos completos para elaborar la selección final de acuerdo a la pertinencia temática siguiendo los criterios enunciados: quedaron 212 artículos.

Para efectuar el análisis se tomaron cinco ejes principales:

1. Vinculación entre normalidad y patología en la elaboración del discurso (especialmente si el análisis de condiciones consideradas patológicas se utiliza como insumo para la construcción de conocimiento sobre desarrollo).

2. Teoría del default²: se la sostiene, se plantea otra lógica para el desarrollo ovárico o no se incluye.

3. Destinos posibles de la determinación: presencia o ausencia de una mirada binaria sobre la determinación sexual (testículo u ovario como únicas opciones).

4. Presencia de conceptos no hegemónicos, nuevas propuestas teóricas, ideas alternativas. Este eje se ajusta a una de las propuestas analíticas de La arqueología del saber, donde Foucault (1979) apuntó a la identificación de márgenes en el discurso como forma de delimitarlo y definirlo.

5. Fecha de publicación.

2. Esa teoría sostiene la idea de que, a diferencia del testículo, el ovario se desarrolla por defecto (default). Se profundiza en este aspecto en la sección 3. 
Se analizaron los ejes propuestos y se valoró su evolución a través del tiempo para estudiar la presencia o no de tendencias o periodizaciones.

\section{Los manuales}

Se seleccionaron los tres manuales más recomendados para el abordaje de la determinación sexual en las carreras públicas de medicina en Argentina. La temática es abordada en distintos espacios de las carreras de acuerdo a cada propuesta curricular. Se revisaron los currículos y se tomó la bibliografía recomendada en 2015 para el espacio curricular donde se aborda principalmente la temática en cada carrera. Los tres manuales más recomendados son:

1. Sadler, T.W. (2012). Langman-Sadler Embriología Médica, 12va edición. Editorial Médica Lipincott.

2. Moore, K.L., Persaud, T.V.N. y Torchia, M.G. (2013). Embriología Clínica (9na edición). Elsevier.

3. Carlson, B. (2014). Embriología humana y Biología del desarrollo (5ta ed.). Elsevier.

En las entrevistas se identifican los mismos textos como los más utilizados. El análisis de los manuales se elaboró sumando a los ejes considerados para la revisión de los artículos el lugar del apartado sobre determinación sexual en el texto (extensión relativa, ubicación en la organización del texto) y el origen del manual.

Se selecciona como enfoque teórico general la propuesta de Ludwik Fleck para indagar en el modo en que la comunidad biomédica contemporánea se organiza en torno a la determinación sexual y la construye a través de su discurso. Las categorías analíticas centrales fueron las de colectivo de pensamiento (en particular en este caso el círculo esotérico ${ }^{3}$ del colectivo de

3. Este término, presente en las traducciones al castellano de la obra de Fleck, refiere al grupo de especialistas que se organiza en torno a una temática determinada. Para un colectivo de pensamiento en particular, el círculo central es el esotérico, en contraposición al círculo periférico compuesto por legos o exotérico. Aquí se focaliza en el círculo esotérico puesto que es el que representa el discurso biomédico en este caso. $\mathrm{Si}$ bien es probable que, dado el significado habitual del término esotérico en castellano, una opción más clara hubiera sido la palabra «endotérico», se mantiene el término original de las traducciones en este artículo. 
pensamiento en torno a la determinación sexual en el período seleccionado) y estilo de pensamiento (Fleck, 1986).

\section{2. ¿QUIÉNES ELABORAN EL DISCURSO BIOMÉDICO SOBRE DETERMINACIÓN SEXUAL ENTRE 1990 Y 2015?: EL CÍRCULO ESOTÉRICO DEL COLECTIVO DE PENSAMIENTO}

Un colectivo de pensamiento es el portador comunitario de un estilo de pensamiento determinado (Fleck, 1986). El círculo esotérico del colectivo de pensamiento sobre determinación sexual durante el período 1990-2015 se mantuvo relativamente estable en cuanto a su organización y al tipo de materiales producidos, y su estructura es, por lo tanto, pasible de ser examinada.

A diferencia de otras temáticas abordadas por la biomedicina, la determinación sexual no pertenece en el campo biomédico a un área única o principal de conocimiento ni de práctica (Audí Parera y Guerrero, 2015). Como otros procesos del desarrollo prenatal, esta temática ha sido abordada clásicamente por la embriología, que en la actualidad se vincula a un grupo relacionado de disciplinas tales como la biología molecular y la genética del desarrollo. Ninguna de las áreas mencionadas corresponde a una única especialidad médica en el campo asistencial. Sin embargo, la repercusión de este discurso en la asistencia es evidente especialmente en especialidades como la endocrinología pediátrica, la pediatría y la cirugía infantil (Audí Parera y Fernández-Cancio 2015; Audí Parera y Guerrero, 2015; GuerreroFernández et al., 2018). Entonces, ¿cómo se organizan estas disciplinas en el círculo esotérico del colectivo de pensamiento que se estructura en torno a esta temática? ¿Qué otros actores participan? ¿Cómo se vinculan?

En el caso de la producción de conocimiento sobre determinación sexual en Argentina en las últimas décadas, el círculo esotérico está compuesto por un núcleo formado por investigadores expertos y una periferia integrada por un grupo de especialistas vinculados a la temática. Los dos centros más importantes del país en la investigación y producción de conocimiento original sobre determinación y diferenciación sexual se encuentran en grandes hospitales pediátricos de la Ciudad de Buenos Aires, vinculados al Consejo Nacional de Investigaciones Científicas y Técnicas (CONICET). Se trata del Hospital Garrahan (hospital público descentralizado) y el CEDIE (Centro de 
Investigaciones Endocrinológicas), un centro asociado al Hospital Gutiérrez, hospital público municipal. Los/as principales investigadores/as de esos centros se identificaron como el centro del círculo esotérico.

Nos conocemos entre nosotros, nos vemos en las reuniones, en los congresos. A veces hace falta mirar algo en una muestra y yo llamo por ejemplo a [nombre de investigadora experta] para que me haga tal gen, lo arreglamos entre nosotros. [...] En las conferencias internacionales se hablan cosas, se cuenta la experiencia, y ahi entre todos decidimos los consensos. (Investigador/a experto/a) ${ }^{4}$

Tienen conexión tanto con personas expertas en otros países como con organizaciones internacionales vinculadas a la producción de conocimiento y toma de decisiones académicas y asistenciales en la temática. Entre estas destacamos la Sociedad Europea de Endocrinología Pediátrica (European Society for Pediatric Endocrinology, ESPE), la Sociedad de Endocrinología Pediátrica (Estados Unidos) (Pediatric Endocrine Society, PES) y la Sociedad Latinoamericana de Endocrinología Pediátrica (SLEP).

Nos conocemos todos. El otro día me llamó [investigador experto británico] porque viene a la Argentina invitado por [nombre de una empresa biofaracéutica], para que organicemos algo. [...] ahora con internet es mucho más fácil también. No somos tantos, nos conocemos, colaboramos. (Médico endocrinólogo pediátrico)

El círculo esotérico del colectivo de pensamiento se completa con especialistas que no son investigadores/as expertos/as en el tema. Es el caso de la docencia en determinación sexual humana, que se da fundamentalmente en la formación médica de grado y, en mucha menor medida, algunos pocos programas de posgrado. Si bien todas las personas entrevistadas son docentes de posgrado en biomedicina, solo dos abordan la determinación sexual normal (en endocrinología y cirugía pediátricas). Con respecto a la formación médica de grado, en las carreras de medicina de Argentina la temática se trabaja clásicamente en una asignatura correspondiente a los primeros años de la carrera, en el contexto del desarrollo prenatal. Se destacan dentro del círculo esotérico los/as docentes de las cátedras de embriología (o equivalentes) de

4. Para los/as investigadores/as expertos/as, que constituyen un colectivo muy pequeño, se decide no especificar el género para preservar el anonimato.

Feminismo/s 38, July 2021, 331-357 
las escuelas de medicina del país porque su posición resulta clave desde la perspectiva de la reproducción del discurso biomédico.

Desde el punto de vista del científico el que está involucrado es el genetista, el biólogo celular, el biólogo molecular esencialmente. [...] Antiguos embriólogos, llamémosles así, que hoy se les llama biólogos del desarrollo. (...)Antes solo los embriólogos nos ocupábamos de esto. [...] lo normal, lo vemos en función de lo que puede servir para los pacientes. De lo normal así puro, aparte de lo científico, seguimos hablando más que nada en embrio. (Investigador/a experto/a)

El otro gran sector de especialistas no expertos/as que integra el círculo esotérico se compone de quienes trabajan en las áreas vinculadas de la medicina asistencial. En relación con las especialidades médicas que abordan la temática, a través de la información obtenida de las entrevistas y los artículos científicos se pudo confirmar y ampliar la estructuración inicial. Como fue referido, la determinación sexual no se corresponde con una especialidad médica única, pero se aborda principalmente en el área asistencial desde la clínica pediátrica, la cirugía pediátrica, la endocrinología pediátrica y la genética médica.

La práctica profesional de estos/as especialistas tiene la particularidad de ser ejercida sobre personas y colectivos del círculo exotérico. Tienen un efecto multiplicador del discurso hacia la periferia del círculo esotérico y sobre actores clave del círculo exotérico como las personas intersex. Son centrales para la consolidación de esta mirada sobre las trayectorias y los cuerpos de muchas personas, con grandes implicancias en la salud pública. Si bien estas especialidades son las centrales, hay otras que pueden implicarse más periféricamente en el caso de la determinación sexual categorizada como normal. Entre ellas, destacamos la ginecología y la urología pediátricas.

Endocrinólogos y ginecólogos infantiles. [...] Cuando nace un chico y nos da duda, está el servicio de ginecología que tiene pediatría, o uro [por urología] [...] Ahora, de la embriología nos ocupamos en la facultad. (Pediatra y docente de embriología)

Cabe aclarar que -en muchos casos- una persona puede participar de varios espacios dentro del círculo esotérico, por ejemplo, ser investigador/a experto/a y docente de embriología, o trabajar en asistencia y docencia. En síntesis, quienes componen el círculo esotérico se desempeñan en la esfera asistencial, de la docencia y/o de la investigación. Las principales especialidades 
médicas que abordan la determinación sexual en el área asistencial comprenden la clínica pediátrica, endocrinología pediátrica, genética médica y cirugía pediátrica.

Un aspecto adicional que se vincula directamente a la organización del colectivo de pensamiento es el modo en que circula la información dentro de este círculo. Una de las características del colectivo biomédico en general, y del colectivo biomédico que se organiza en torno a la determinación sexual en particular, es la vasta circulación de determinada información que tiende a la construcción de discursos hegemónicos globales. Los/as especialistas destacaron que la circulación de información biomédica dentro del centro del círculo esotérico de este colectivo de pensamiento, especialmente en los últimos años, se efectúa en gran medida a través del acceso a artículos en medios electrónicos. A diferencia de lo que ocurre en otras áreas, relatan que en biomedicina los artículos suelen ser obtenidos por separado y la lectura de revistas completas es mucho menos habitual. En este sentido, se consultó con los/as referentes del centro del círculo esotérico el modo en que seleccionan los artículos. Un investigador destacó la enorme importancia de los sistemas que proveen en forma automática recomendaciones de lectura a partir de algoritmos que toman en cuenta los perfiles y selecciones previas de cada usuario, y los señaló como la forma que más utiliza para seleccionar los artículos que leerá.

... de cada 100 artículos que me interesaban yo 95 los encontraba por Pubmed. Hoy eso debe haber bajado a menos de la mitad. [...] Me llegan, me llegan, me llegan. Entonces: ah, me interesó. Entonces como me interesó me meto y encuentro otro, y miro otro. (Investigador/a experto/a)

Estos sistemas pertenecen a grandes empresas de gestión de información científica, y en ocasiones las suscripciones son provistas por empresas privadas, como laboratorios de productos biomédicos. Sin embargo, la persona entrevistada destacaba la practicidad de estas recomendaciones de lectura sin problematizar el posible sesgo en el acceso y circulación del conocimiento a partir de la extensión de estos sistemas.

Otros modos de acceso destacados fueron las búsquedas directas en Pubmed y las recomendaciones de colegas dentro del círculo esotérico. Se observó una tendencia creciente en la utilización de los mencionados sistemas de recomendación como formas de selección de artículos en los últimos

Feminismo/s 38, July 2021, 331-357 
años, en detrimento de las búsquedas directas. Algunos/as especialistas destacaron la utilización de un híbrido entre la búsqueda directa y la utilización de algoritmos informáticos: el sistema de recomendaciones de lectura de Pubmed. A partir de las búsquedas efectuadas por un usuario, Pubmed sugiere artículos relacionados en la misma pantalla en que se muestran los resultados. Así, los sistemas automatizados van ganando terreno.

Otro punto a tener en cuenta es el acceso a los textos completos. En este sentido, dos personas mencionaron como ventaja que la industria farmacéutica y biotecnológica facilita suscripciones a determinadas plataformas que permiten obtener recomendaciones de lectura y acceso a los textos completos de los artículos sugeridos. Asimismo, ofrece facilidades para asistir a reuniones de difusión y discusión en la temática, donde -como se verá en el próximo párrafo- también se comparte información. En este punto, el complejo biomédico-industrial-financiero aparece como un actor privilegiado, por lo poderoso y lo no percibido, siendo un elemento fundamental en la configuración de la biopolítica contemporánea (Rose, 2012). No es objeto de este trabajo profundizar en su funcionamiento en torno a este discurso particular, pero resulta relevante como foco de futuras investigaciones.

Al indagar sobre otros modos de comunicar y acceder a información dentro del círculo esotérico, se mencionaron las reuniones científicas y, secundariamente, los foros profesionales u otros sistemas de comunicación online vinculados con sociedades científicas u otras organizaciones de especialistas. En algunos casos, a partir de esas interacciones se elaboran publicaciones consensuadas, como ocurrió con la producción del Consenso de Chicago (Hughes et al., 2006). Se trata de un documento elaborado a partir de una reunión de expertos ocurrida en la ciudad de Chicago en 2005. Este consenso, aún en vigencia, modificó la clasificación de un grupo de condiciones caracterizadas por un desarrollo sexual que la biomedicina considera diferente al habitual ${ }^{5}$ incorporando aspectos no valorados previamente y erradicando la categoría «pseudohermafroditismo» (Delimata et al., 2018).

5. En este sentido, el historiador y activista intersex Mauro Cabral (2003) se diferencia de la mirada biomédica y sostiene que la intersexualidad no es una enfermedad sino que constituye una condición de no conformidad física con criterios culturalmente definidos de normalidad corporal. 
Si bien el documento se enfoca en aspectos considerados patológicos de la diferenciación, está producido por el mismo colectivo de pensamiento que elabora el discurso sobre determinación sexual. Como se mencionó en el apartado anterior, aquello considerado patológico es tomado como insumo en la producción de este discurso sobre normalidad.

[...] en general las preguntas suelen generarse a partir de observaciones de anomalías del desarrollo sexual. Entonces eso es lo que lleva a la pregunta. Después por supuesto esa pregunta que surgió de una observación clínica la toman grupos básicos que ya tienen conocimientos y qué se yo qué y eso por allí los orienta. Y algunas veces por ahí los grupos básicos sin necesidad de esta pregunta ya están en una línea de investigación y generan conocimiento nuevo y ese conocimiento nuevo después sirve para entender algo que hasta ese momento no se explicaba. Esta es la ida y vuelta de esto que se llama investigación traslacional hoy en día. (Investigador/a experto/a)

Nadie explicitó algún impacto de fuentes informales o creencias personales. Sin embargo, los/as dos investigadores/as expertos/as y un/a especialista no experto/a introdujeron la existencia de organizaciones de familias y -muy especialmente-pacientes intersex o que fueron diagnosticados con alguna de las llamadas anomalías de la diferenciación sexual (DSD ${ }^{6}$ ). Si bien en las entrevistas se tendía a caracterizarlos como actores demandantes-especialmente en relación con la crítica en torno a las intervenciones quirúrgicas sobre sus genitales para normalizarlos durante la infancia- no se mencionó su posible incidencia en la producción del discurso sobre el proceso de la determinación sexual. Sin embargo, en una entrevista se mencionó la conveniencia de instalar en las escuelas baños unisex para no «forzar a los niños»-en principio intersex pero luego en general a todos los niños- a optar por un baño para hombres u otro para mujeres. De este modo, aparecen elementos en el relato de que enuncian en mayor o menor medida el impacto social por fuera del círculo esotérico del discurso biomédico en torno a la determinación sexual.

6. Las anomalías del desarrollo sexual (DSD, del inglés Disorders of Sexual Development) son un grupo heterogéneo de condiciones que la biomedicina engloba como anomalías derivadas de alteraciones en este proceso del desarrollo (Guercio et al., 2010). En los últimos años se ha sugerido el término menos patologizante de desarrollo sexual diferente (Different Sex Development) (Audí Parera y Fernández-Cancio, 2015).

Feminismo/s 38, July 2021, 331-357 
[...] trabajar sobre la aceptación de las diferencias. Hay mucho para trabajar porque habría que trabajar en las escuelas muchísimo. [...] Para la comunidad médica, las escuelas, para insertar digamos en la sociedad que esta estigmatización de nena o varón no está bien, que en realidad todos nosotros tenemos componentes masculinos y femeninos. (Investigador/a experto/a)

Asimismo, si bien como se ha señalado más arriba no se encontró referencia explícita en torno a la incidencia de otros actores sociales sobre el círculo esotérico, estos comentarios visibilizan su presencia y permiten inferir cierta noción -más o menos consciente, mejor o peor valorada- de su impacto. Este impacto, que escapa a la circulación de conocimiento dentro del círculo esotérico que es objeto de este trabajo, sería multidireccional. Por un lado, comprendería la acción de diversos actores que no pertenecen a la academia biomédica interactuando con el círculo esotérico: han sido mencionados los colectivos de personas intersex pero también podrían incidir otros grupos como la industria farmacéutica/biotecnológica y sectores religiosos. Por otro lado, actuando sobre el círculo esotérico, la circulación de conocimiento desde el exotérico podría incidir en la normalización de los cuerpos sexuados y de ese modo en la construcción de ciudadanía biológica en términos de Rose (2012). El aspecto multidireccional para estas circulaciones de conocimiento se complementaría, potencialmente, con la idea aportada por Fleck (1986) de que los mismos individuos pertenecen a varios colectivos de pensamiento en forma simultánea. Así, una persona experta en determinación sexual podría pertenecer también, por ejemplo, a un grupo católico o a un colectivo feminista. Estas dimensiones podrían ser objeto de futuras investigaciones.

\section{LAS PRODUCCIONES ESCRITAS}

Las principales producciones que reconocieron dentro del círculo esotérico sobre determinación sexual las personas entrevistadas son, tal como describió Fleck (1986), los artículos científicos y los manuales de estudio. Los manuales de estudio contienen un recorte del discurso elaborado por personas que -en muchos casos- no son referentes del círculo esotérico. Ese recorte reconoce como fuente los artículos científicos y cumple un rol central en la reproducción del discurso en la formación médica. 
Los consensos producidos en reuniones de expertos/as, si bien son publicados también bajo el formato de artículos para revistas científicas, son un material particular. El único publicado con vinculación directa a la determinación sexual humana entre 1990 y 2015 es el mencionado Consenso de Chicago (Hughes et al., 2006). En la producción de los artículos se observa como la herramienta más frecuentemente utilizada una combinación entre datos clínicos, utilización de la comparación -y en ocasiones extrapolaciónde datos obtenidos a partir del estudio de otras especies, y distintas técnicas de laboratorio enfocadas en aspectos moleculares. En el caso de los artículos que aportan nuevos datos primarios, ese conocimiento se presenta cotejado con una revisión de la bibliografía biomédica en torno al tema. Otros artículos se presentan como revisiones o actualizaciones del estado de la cuestión desarrolladas a partir de selecciones bibliográficas. Se indexan como review 78 de los 212 artículos analizados. Al revisar cada uno de ellos encontramos gran variabilidad en la calidad de las revisiones y, en muchos casos, no hemos podido valorarlas por la ausencia total o parcial de datos metodológicos. Esas selecciones contienen artículos producidos, en su mayoría, a partir de la combinación de las herramientas antes citadas. En forma excepcional algunas publicaciones aportan otros enfoques, de corte más epistemológico, como la contribución de las miradas feministas (Fausto-Sterling, 2015).

Hay una diferencia a lo largo del período analizado en la utilización de datos provenientes de otras especies. La estrategia de la embriología comparada, rama de la embriología que estudia la ontogenia en términos comparativos entre especies, es un abordaje clásico en el estudio biomédico del desarrollo prenatal. Dada la complejidad ética en la experimentación con embriones humanos, obtener datos de otras especies y luego intentar cotejarlas con lo que ocurre en nuestra especie ha sido una opción muy utilizada. En el caso particular de la determinación sexual ha ocurrido lo contrario en la detección de varias de las moléculas involucradas. Es decir, en lugar de ir desde los animales hacia las personas, la identificación de determinadas dianas moleculares en nuestra especie permitió intentar establecer modelos de estudio en animales. A partir del estudio de personas que la biomedicina identifica como portadoras de determinados fallos en el proceso de determinación sexual, se identificaron moléculas involucradas que luego se buscaron en otros mamíferos (Goodfellow y Lovel-Badge, 1993; Ostrer, 2001).

Feminismo/s 38, July 2021, 331-357 
Sin embargo, en este proceso se han observado muchas diferencias entre especies, aun en aquellas que tienen mecanismos de desarrollo similares para otros órganos. Estas diferencias han sido señaladas en todo el período analizado pero, en especial en los últimos 10 años, la necesidad de obtener datos provenientes de nuestra especie para conocer en detalle la determinación sexual humana se ha consolidado como parte del estilo de pensamiento (Boyer et al., 2006; Valenzuela, 2008).

Al observar las formas de construir conocimiento durante este período, se destacan dos dimensiones en la articulación de los datos citados: la vinculación entre lo normal y lo patológico, y la utilización de nuevas tecnologías para análisis moleculares.

\section{a. Lo patológico en la construcción del discurso sobre determinación sexual normal}

Se observó ampliamente en los materiales analizados la utilización de elementos considerados patológicos como insumos para la producción del discurso sobre normalidad. Estos elementos patológicos pueden ser de ocurrencia no intencional -como la recopilación de datos a partir del estudio de personas categorizadas como portadoras de alteraciones en el desarrollo genital- o bien pueden estar producidos deliberadamente a través de la introducción de modificaciones en modelos animales a fin de analizar las funciones de una molécula particular.

Sobre el estudio de personas caracterizadas como portadoras de alteraciones en el desarrollo genital, las ventajas que fueran señaladas por Canguilhem (1971) son aprovechadas -y destacadas- por los investigadores. Esto es utilizado generalmente para constatar presuposiciones sobre el significado biológico de determinadas moléculas. Entre 1990 y 2015 han sido de particular interés para este colectivo de pensamiento los casos de sex-reversal (inversión de sexo). Estas personas tienen una constitución cromosómica esperada por la biomedicina para un sexo determinado pero los genitales esperados para el otro sexo, por supuesto desde una concepción binaria de esa categoría. En otras palabras, se trata de personas intersex con constitución cromosómica XX que tienen genitales que la biomedicina considera característicos de los machos o, a la inversa, alguien con constitución 
cromosómica XY que porta genitales que este colectivo espera encontrar en quienes entiende como hembras.

La utilización de esta herramienta se evidencia con claridad en la década de los '90, donde se estudió ampliamente la correlación entre la presencia de un gen particular y el desarrollo testicular. Se postulaba, por ejemplo, como se ha consignado en este trabajo, que el gen SRY era condición necesaria y suficiente para la producción del desarrollo testicular. En machos considerados normales el gen SRY es parte del cromosoma Y. Entonces, se presumía que las personas con constitución cromosómica XY pero sin desarrollo testicular portan una condición patológica de carencia de gen SRY en su cromosoma Y. A fin de confirmar este postulado se investigó la presencia de ese gen en personas con constitución cromosómica XY y sin desarrollo testicular, con resultados contrapuestos. En algunos casos se observó correlación entre la ausencia de gen SRY funcional en el cromosoma Y de personas con constitución cromosómica XY, y la ausencia de testículos (Farah et al., 1991; Ryner y Swain, 1995). Del mismo modo, resultados contrapuestos se obtienen a través del análisis de la presencia (señalada como patológica) del gen SRY en personas con constitución cromosómica XX y tejido testicular. Proliferan en esta época los reportes de casos en los que, a través del estudio genético de personas con condiciones consideradas patológicas por la biomedicina, se pretende aportar solidez al discurso sobre normalidad (Brown et al., 1998). Es interesante destacar que si bien en algunas situaciones se constata esa correlación y se utiliza para reafirmar el discurso de ese momento, un grupo de personas con esa constitución (XY pero sin gen SRY) tienen testículos a pesar de no contar con ese gen. En muchos artículos este grupo es consignado como resultado marginal pero llamativamente en la primera década no se problematiza masivamente su ocurrencia en el estilo de pensamiento, contraria al modelo hegemónico de determinación en ese período. Es decir, en estos primeros 10-15 años del período analizado, con una concepción general más lineal y determinista del proceso de determinación sexual, cuando la utilización de lo patológico contradice al menos parcialmente la teoría hegemónica, ese dato es registrado pero ignorado por gran parte del colectivo de pensamiento.

Además de las situaciones mencionadas, donde lo patológico aparece como una consecuencia fortuita de una desviación del curso normal, se

Feminismo/s 38, July 2021, 331-357 
observó en el período analizado la utilización de errores inducidos en el proceso de determinación en modelos animales como método de construcción de conocimiento. La estrategia más utilizada es la producción de animales mutantes para genes determinados en los que se elimina un gen específico. De ese modo, al observar la determinación sexual de ese animal, se pretende inferir la acción del producto de ese gen al observar el resultado de su carencia. En los desarrollos considerados patológicos no se produce la vuelta al estado normal -descripto por Canguilhem (1971) como central en la constatación de los mecanismos fisiológicos-. Estos experimentos funcionan como una alternativa para obtener artificialmente a través de la manipulación genética de animales esas constataciones (Graves, 1997; Josso y Rey, 1996; Lim y Hawkins, 1998; Swain y Lovell-Badge, 1997). Como se ha comentado antes, en los últimos 10 años disminuyó la utilización de estas estrategias en reemplazo de los estudios en humanos.

Al examinar el modo en que opera la vinculación con lo patológico en la estructuración del discurso sobre normalidad en la determinación sexual, se observa que, como propuso Canguilhem (1971), el estudio científico de aquello considerado casos patológicos resulta clave en la investigación de las leyes del estado normal. Al tratar de desentrañar esas leyes -la normatividad vital de la determinación sexual-aparece con claridad en este objeto la búsqueda de las normas de lo patológico. Esta condición no es la ausencia de normas, como dice Canguilhem, sino que su lógica de funcionamiento es diferente a la del estado normal y condiciona un funcionamiento comparativamente inferior. En el caso de la determinación sexual esa lógica es utilizada, como se consignó en los párrafos precedentes, para deducir a su vez leyes fisiológicas. En este sentido resulta importante destacar que la mayor parte del círculo esotérico de este colectivo de pensamiento compone también aquel que se organiza en torno al discurso sobre las DSD.

\section{b. La utilización de tecnologías de laboratorio}

La visualización de estructuras microscópicas ha tenido gran relevancia en la construcción del discurso sobre determinación sexual en la historia occidental. Entre los siglos XVII y XIX las técnicas de microscopía tuvieron un papel central en la descripción de testículos, ovarios, óvulos y espermatozoides 
(Mittwoch, 2013). En el siglo XX las técnicas de visualización se centraron en estructuras sub-celulares, y en ese contexto se produjeron la descripción de los cromosomas sexuales y el nacimiento de la genética clásica (Delgado Echeverría, 2000; Maienschein, 1984; Mittwoch, 2013). Hacia finales del siglo XX las tecnologías disponibles posibilitaron hacer foco en estructuras cada vez más pequeñas. En 1990 se consolidó esta búsqueda en el inicio del Proyecto Genoma Humano, que se proponía detallar la composición del ADN de nuestra especie. En ese mismo año, la descripción del gen SRY irrumpió como la solución al enigma de la determinación sexual con un enfoque determinista, inscribiendo la teoría del default en el modelo de la genética clásica. Esto significa que, en ausencia de determinados atributos que generarían testículos -centralizados en el producto del gen SRY a partir de 1990-, las gónadas embrionarias se convierten por defecto en ovarios (Blyth y Duckett, 1991; Brown et al., 1998; Knower et al., 2003). La idea de que un órgano se desarrolle de este modo, solo por la ausencia de un elemento y sin determinantes moleculares que organicen su estructuración, en el marco de los discursos biomédicos en el período analizado era impensable en 1990 para cualquier otro órgano del cuerpo humano. Pero en determinación sexual no solo fue posible, sino que se instituyó en la concepción hegemónica.

En la primera década después de la presentación del gen SRY, se intentó describir su mecanismo de acción y el discurso se focalizó en cuestiones moleculares. Las tecnologías de laboratorio eran ya herramientas imprescindibles en la construcción del discurso.

A partir de 2000 se multiplicaron los artículos que apuntaban a las tecnologías de laboratorio (fundamentalmente vinculadas con genética y genómica) como fuentes, combinadas o no con datos clínicos -sobre todo relacionados con las DSD-y/o con enfoque de embriología comparada. Continuó la hegemonía de los genes en el discurso, pero fueron apareciendo elementos adicionales como la importancia de las dosis de productos del gen (ya no solo su presencia o ausencia, activación o inhibición) en el momento adecuado (timing) (Gross, 2006; Polanco y Koopman, 2006), la importancia del procesamiento postranscripcional ${ }^{7}$ y la ubicación celular de las moléculas

7. El procesamiento postranscripcional comprende una serie de mecanismos que operan sobre la molécula resultante de la transcripción de un gen determinado, modificándola.

Feminismo/s 38, July 2021, 331-357 
(Li et al., 2001), y los mecanismos epigenéticos ${ }^{8}$ (Bouayed Abdelmoula et al., 2003). En la última década del período analizado, si bien la descripción de genes involucrados en el discurso sobre determinación sexual se multiplicó, la explicación se profundizó aún más en términos moleculares y se añadieron otros sistemas de regulación al de los genes, que continuó siendo hegemónico. Por ejemplo, aparecieron como posiblemente relevantes en la determinación sexual sistemas de regulación molecular de descripción reciente como el de los $\mathrm{ARNpi}^{9}$ (Huang et al., 2014). Estos nuevos tipos de análisis y las descripciones de mecanismos adicionales implicaron el uso de tecnologías específicas que se fueron desarrollando en los últimos años.

En algunas entrevistas y en numerosos artículos -especialmente entre 2010 y 2015- se hace referencia a los avances recientes en términos de tecnologías genéticas y genómicas, y al modo en que posibilitan descripciones más precisas de procesos y moléculas involucradas en materia de determinación sexual (Bashamboo y Mcelreavey, 2015; Eggers y Sinclair, 2012; Wilhelm, Yang y Thomas, 2013; entre otros). Se señala, asimismo, que estas tecnologías aún no han sido suficientemente explotadas y representan herramientas que posibilitarán nuevos horizontes -moleculares- en la caracterización de la determinación sexual.

La utilización de tecnologías cada vez más complejas orientadas a la visualización de estructuras cada vez más pequeñas es notable, pero no es el único cambio. También esas tecnologías son utilizadas para describir nuevos sistemas de regulación de la determinación sexual que permiten

En algunos casos eso permite obtener diferentes productos - con distintas posibles acciones- partiendo de un mismo gen.

8. Los mecanismos epigenéticos son las modificaciones que puede sufrir un gen sin afectar su secuencia de nucleótidos. Tienen la capacidad de modificar la funcionalidad de los genes. Por ejemplo pueden hacer que, a pesar de que un gen esté presente en un organismo determinado, no pueda transcribirse. Es decir -en este ejemplo- las modificaciones epigenéticas que operan en un gen condicionan su funcionalidad de tal manera que pueden provocar que un gen -aun si contiene la secuencia esperada-esté presente en un organismo pero no pueda ser utilizado (lo silencian).

9. Los ARNpi (piwi-interacting ARNs) son un grupo de moléculas de reciente descripción. Corresponden a pequeñas moléculas de ARN que no codifican proteínas y se postulan como cruciales en el desarrollo temprano, la regulación epigenética, la gametogénesis, la regulación del ciclo celular, y el silenciamiento de elementos transposables y algunos genes que sí codifican proteínas.

Feminismo/s 38, July 2021, 331-357 
analizarla como un proceso más plástico e incluso dinámico, abriendo el juego a caracterizar desde lo molecular un sexo no tan fijo ni tan binario (Hadid, 2018). Sin embargo, estas nuevas descripciones no aparecen en los manuales analizados.

Otro aspecto a considerar consiste en el modo en que circula la información a través del colectivo de pensamiento. Dentro del centro del círculo esotérico, de acuerdo con los datos obtenidos, la circulación de conocimiento se da a través de la lectura de artículos científicos y, en menor medida, por comunicación entre colegas y en reuniones científicas. A medida que nos acercamos a la periferia del círculo esotérico, los manuales se van consolidando como el principal material utilizado. En palabras de una persona entrevistada (especialista en cirugía pediátrica ginecológica) se utilizan los «libros de embriología» para abordar la determinación sexual tanto en los ateneos clínico-quirúrgicos como en la formación de médicos/as residentes en cirugía. Lo mismo ocurre en la formación de grado. Este material resulta central por su capacidad de multiplicar el discurso a través de la educación médica.

\section{CONSIDERACIONES FINALES}

Este artículo indaga en los modos en que la biomedicina contemporánea organiza su discurso sobre la determinación sexual. Se destaca la enunciación del discurso como la resultante del descubrimiento de una verdad natural por un observador objetivo. Sin embargo, como ocurre con otras construcciones colectivas, se observa que esta perspectiva aparentemente independiente de la historia y de las cosmovisiones no es tal. Esto se puede ver con claridad al estudiar cómo este colectivo de pensamiento utiliza una combinación de herramientas con presunta tradición de objetividad como son las tecnologías de laboratorio -especialmente aquellas orientadas a la observación de estructuras sub-celulares-, la utilización de datos obtenidos de la clínica, y el empleo de información obtenida a partir de otras especies, para sostener la teoría del default. Esta idea resulta impensada en la misma época para explicar el desarrollo de cualquier otro órgano humano. Sin embargo, para definir la existencia de la diferencia sexual como hecho natural y explicar su génesis es hegemónica. El ovario aparece como resultante por defecto a 
partir de una carencia, y el testículo es la consecuencia de la acción de un atributo positivo que permite completar el desarrollo. Sostener este tipo de explicaciones en la actualidad refuerza una concepción patriarcal fuertemente arraigada en la tradición de la ciencia biomédica y, por supuesto, se explica justamente por la presencia de este enfoque.

Esta visión se hace aún más evidente en los manuales de estudio. Este material contiene un recorte del discurso especializado elaborado especialmente para su reproducción a través de la formación médica. Si bien no es objeto de este artículo profundizar en el contenido del discurso, a fines de complementar la reflexión, destacamos que la mirada del colectivo de pensamiento en relación con un modelo de sexos binario, fijo y jerarquizado (heteropatriarcal, en definitiva) se hace especialmente presente en la elaboración de esta selección que destina a la difusión y reproducción del discurso dentro de la comunidad biomédica. De ese modo, aparece una síntesis que refleja la visión clásica sobre la determinación sexual humana-vinculada a la teoría del default- en detrimento de las nuevas miradas presentes en el discurso especializado en los últimos años del período analizado. No aparece en los manuales de estudio analizados la posibilidad de una determinación sexual no binaria, dinámica, ni se describe el desarrollo de las hembras como activo y positivo en términos moleculares.

Retomando el aporte que hace Fleck (1986) al considerar fundacional para el modo en que la biomedicina construye sus discursos la falta de reflexión crítica sobre su epistemología, a la luz de los resultados de esta investigación se observa que estos análisis resultan necesarios para la modificación de las prácticas biomédicas -incluyendo entre ellas al propio discurso-. En el caso particular del discurso biomédico sobre determinación sexual humana, la circulación es en dos sentidos. Así como las concepciones del colectivo de pensamiento sobre el dispositivo sexo-género resultan centrales para la consolidación del conocimiento, el propio discurso y otras prácticas biomédicas que se apoyan en él tienen implicancias sobre las concepciones y las acciones de diversos colectivos en torno a los cuerpos, los géneros, y los derechos sexuales y reproductivos. Expresa y sostiene relaciones de poder y estructuración de jerarquías. Perpetúa condiciones opresivas y normalizadoras, y afecta especialmente a las personas que deben decidir sobre sus cuerpos o los de sus hijos, pero también los modos de concebir

Feminismo/s 38, July 2021, 331-357 
la diferencia sexual, las corporalidades y las diversidades. En definitiva, el impacto del discurso biomédico sobre la determinación sexual en la estructuración de la verdad biomédica sobre el cuerpo normal tiene efectos para la salud colectiva, y está en construcción recíproca con las prácticas y representaciones no biomédicas.

Reconocer que el discurso biomédico no es la revelación de una verdad única y objetiva, y analizar su construcción, permite avanzar en el conocimiento tanto de las condiciones que lo sostienen como de las que lo delinean y restringen. Así, las relaciones de fuerzas, las lógicas subyacentes y la construcción de verdad se evidencian. Esto facilita ampliar las fronteras del propio discurso para abrir las posibilidades del colectivo de pensamiento a examinar nuevas posibilidades. Se destaca la falta de problematización de esta construcción como objeto epistemológico considerando sus implicancias en la estructuración de la verdad biomédica sobre los cuerpos normales. Este punto es central para pensar futuras acciones en investigación y política pública, como pueden ser la introducción de la reflexión epistemológica en la formación médica y la capacitación en género como parte de los planes de estudio de las carreras de grado en medicina. Consideramos que la planificación estratégica de recursos humanos en salud tanto para la asistencia como para la docencia, divulgación e investigación, debe incluir como una más de sus dimensiones de acción la formación en diversidad corporal y de género, no binarismo de género y perspectiva de derechos humanos.

\section{REFERENCIAS BIBLIOGRÁFICAS}

Audí Parera, L. y Guerrero, J. (2015). III Curso de Actualización sobre Desarrollo Sexual Diferente (DSD) (no HSC). Curso de Avances de la Sociedad Española de Endocrinología Pediátrica (SEEP). Revista Española Endocrinología Pediátrica, 6(2), 4-6. https://doi.org/fg77

Audí Parera, L. y Fernández-Cancio, M. (2015). La atención integral a los DSDs desde la perspectiva médica. Terminologías, registros y proyectos colaborativos. Revista Española Endocrinología Pediátrica, 6(2), 15-20. https://doi. $\operatorname{org} / \mathrm{fg} 78$

Bashamboo, A. y Mcelreavey, K. (2015). Human sex-determination and disorders of sex-development (DSD). Seminars in cell \& developmental biology, 45, 77-83. https://doi.org/10.1016/j.semcdb.2015.10.030

Feminismo/s 38, July 2021, 331-357 
Blyth, B. y Duckett, J.Jr. (1991). Gonadal differentiation: a review of the physiological process and influencing factors based on recent experimental evidence. The Journal of Urology, 145(4), 689-694. https://doi.org/10.1016/ S0022-5347(17)38426-4

Bouayed Abdelmoula, N., Portnoi, M.F., Keskes, L., Recan, D., Bahloul, A., Boudawara, T., Saad, A. y Rebai, T. (2003). Skewed X-chromosome inactivation pattern in SRY positive XX maleness: a case report and review of literature. Annales de Génétique, 46(1), 11-18. https://doi.org/10.1016/ S0003-3995(03)00011-X

Boyer, A., Pilon, N., Raiwet, D. L., Lussier, J. G. y Silversides, D. W. (2006). Human and pig SRY 5' flanking sequences can direct reporter transgene expression to the genital ridge and to migrating neural crest cells. Developmental Dynamics, 235(3), 623-632. https://doi.org/10.1002/dvdy.20670

Brown, S., Yu, C.C., Lanzano, P., Heller, D., Thomas, L., Warburton, D., Kitajewski, J. y Stadtmauer, L. (1998). A de novo mutation (Gln2stop) at the 5' end of the SRY gene leads to sex reversal with partial ovarian function. American Journal of Human Genetics, 62(1), 189-192. https://doi.org/10.1086/301684

Cabral, M. (2003). Pensar la intersexualidad, hoy. En D. Maffía (Ed.), Sexualidades migrantes (pp. 117-126). Feminaria Editora.

Canguilhem, G. (1971). Lo normal y lo patológico. Siglo XXI Argentina S.A.

David, M. y Sutton, C. D. (2004). Social Research: The Basics. Sage.

Delgado Echeverría, I. (2000). Nettie Maria Stevens y la función de los cromosomas sexuales. Cronos: Cuadernos valencianos de historia de la medicina y de la ciencia, 3(2), 239-272. http://hdl.handle.net/10261/101257

Delimata, N., Simmonds, M., O’Brien, M., Davis, G., Auchus, R. y Lin-Su, K. (2018). Evaluando el término trastornos del desarrollo sexual: un debate multidisciplinario / Evaluating the Term 'Disorders of Sex Development': A Multidisciplinary Debate. Medicina Social, 11(3), 95-105.

Eggers, S. y Sinclair, A. (2012). Mammalian sex determination-insights from humans and mice. Chromosome research, 20(1), 215-238. https://doi. org/10.1007/s10577-012-9274-3

Farah, S.B., Garmes, H.M., Cavalcanti, D.P., Mello, M.P., Porrelli, R.N., Ramos, C.F. y Sartorato, E.L. (1991). Use of Y-chromosome-specific DNA probes to evaluate an XX male. Brazilian Journal of Medical and Biological Research, 24(2), 149-256. 
Determinación sexual: ¿cómo estructura la biomedicina contemporánea su discurso sobre la génesis de la diferencia? Un estudio con foco en Argentina

Fausto-Sterling, A. (2015). Intersex: concept of multiple sexes is not new. Nature, 519 (7543), 291. https://doi.org/10.1038/519291e

Fernández-Altuna, M., Martínez del Prado, A., Arriarán Rodríguez, E., Gutiérrez Rayón, D., Toriz Castillo, H.A. y Lifshitz Guinzberg, A. (2016). Uso de los MeSH: una guía práctica. Investigación en educación médica, 5(20), 220-229. https://doi.org/10.1016/j.riem.2016.02.004

Fleck, L. (1986). La génesis y el desarrollo de un hecho científico: Introducción a la teoría del estilo de pensamiento y del colectivo de pensamiento. Alianza Editorial.

Foucault, M. (1979). La arqueología del saber. Siglo XXI editores.

Foucault, M. (1998). Historia de la sexualidad. I La voluntad de saber. Siglo XXI editores.

Foucault, M. (2003). El nacimiento de la clínica: una arqueología de la mirada médica. Siglo XXI Editores.

Gilbert, S. (2010). Developmental biology (9na ed.). Sinauer.

Goodfellow, P. N. y Lovell-Badge, R. (1993). SRY and sex determination in mammals. Annual reviews of Genetics, 27, 71-92. https://doi.org/10.1146/annurev. ge.27.120193.000443

Graves, J.A. (1997). Two uses for old SOX. Nature Genetics, 16(2), 114-115. https:// doi.org/10.1038/ng0697-114

Gross, L. (2006). Male or female? It depends on the dose. PLoS Biology, 4(6), e211.https://doi.org/10.1371/journal.pbio.0040211

Guercio, G., Constanzo, M., Berensztein, E., Bailez, M., López, J. C., Durán, V., Ongaro, L., Belgorosky, A. y Rivarola, M. (2010). Anomalías de la diferenciación sexual (DSD). Enfoque pediátrico. Revista medicina Infantil, XVII (2), 184-189. https://www.medicinainfantil.org.ar/images/stories/volumen/2010/ xvii_2_170.pdf

Guerrero-Fernández, J., Azcona San Julián, C., Barreiro Conde, J., Bermúdez de la Vega, J.A., Carcavilla Urquí, A., Castaño González, L.A., Martos Tello, J.M., Rodríguez Esteves, A., Yeste Fernández, D., Martínez Martínez, L., Martínez-Urrutia, M.J., Mora Palma, C. y Audí Parera, L. (2018). Guía de actuación en las anomalías de la diferenciación sexual (ADS) / desarrollo sexual diferente (DSD). Anales de Pediatría, 89(5), 315.el-315.e19. https://doi. org/10.1016/j.anpedi.2018.06.009

Hadid, L. (2018). La genética en el discurso biomédico contemporáneo sobre determinación sexual. RevIISE, 12, 135-145. 
Determinación sexual: ¿cómo estructura la biomedicina contemporánea su discurso sobre la génesis de la diferencia? Un estudio con foco en Argentina

Huang, Y., Bai J.Y. y Ren H.T. (2014). PiRNAs biogenesis and its functions. Russian Journal of Bioorganic Chemistry, 40(3), 320-326. https://doi.org/10.1134/ S1068162014030169

Hughes, I., Houk, C., Ahmed, S.F., Lee, P.A., Lawson Wilkins Pediatric Endocrine Society (LWPES) y European Society for Paediatric Endocrinology (ESPE) (2006). Consensus statement on management of intersex disorders. Archives of Disease on Childhood, 91(7), 554-563. https://doi.org/10.1136/ adc.2006.098319

Josso, N. y Rey, R. (1996). Genetic mechanisms of sex differentiation. Current Opinion in Pediatrics, 8(4), 396-400. https://doi. org/10.1097/00008480-199608000-00017

Knower, K.C., Kelly, S. y Harley, V.R. (2003). Turning on the male--SRY, SOX9 and sex determination in mammals. Cytogenetic and Genome Research, 3(4), 185-198. https://doi.org/10.1159/000074336

Li, B., Zhang, W., Chan, G., Jancso-Radek, A., Liu, S. y Weiss, M.A. (2001). Human sex reversal due to impaired nuclear localization of SRY. A clinical correlation. The Journal of Biological Chemestry, 276(49), 46480-46484. https://doi.org/10.1074/jbc.C100388200

Lim, H.N. y Hawkins, J. (1998). Genetic control of gonadal differentiation. Bailliere's Clinical Endocrinology and Metabolism, 12(1), 1-16. https://doi. org/10.1016/S0950-351X(98)80410-2

Maienschein, J. (1984). What determines sex? A study of converging approaches: 1880-1916. Isis: An international review devoted to the history of science and its cultural influences, 75(278), 457-480. https://doi.org/10.1086/353567

Mc Laren, A. (1990). What makes a man a man? Nature, 346(6281), 216-217. https://doi.org/10.1038/346216a0

Mittwoch, U. (2013). The study of sex determination. EMBO reports, 14(7), 588592. https://doi.org/10.1038/embor.2013.84

Nagai, K. (1996). Molecular basis governing primary sex in mammals. The Japanese Journal of Human Genetics, 41(4), 363-379. https://doi.org/10.1007/ BF01876327

Nordqvist, K. (1995). Sex differentiation -- gonadogenesis and novel genes. The International Journal of Developmental Biology, 39(5), 727-736.

John Hopkins University (2017, marzo). Número MIM: 480000 - OMIM (Online Mendelian Inheritance in Man). https://www.omim.org/entry/480000\#title. 
Ostrer, H. (2001). Identifying Genes for Male Sex Determination in Humans. Journal of Experimental Zoology, 290, 567-573. https://doi.org/10.1002/ jez.1107

Polanco, J.C. y Koopman, P. (2006). Sry and the hesitant beginnings of male development. Developmental Biology, 302(1), 13-24. https://doi.org/10.1016/j. ydbio.2006.08.049

Ronfani, L. y Bianchi, M.E. (2004). Molecular mechanisms in male determination and germ cell differentiation. Cellular and Molecular Life Sciences, 61(15), 1907-1925. https://doi.org/10.1007/s00018-004-4034-y

Rose, N. (2012). Políticas de la vida: Biomedicina, poder y subjetividad en el siglo $X X I$. UNIPE.

Ryner, L.C. y Swain, A. (1995). Sex in the '90s. Cell, 81, 483-493. https://doi. org/10.1016/0092-8674(95)90069-1

Safatle, V. (2011). O que é uma normatividade vital? Saúde e doença a partir de Georges Canguilhem. Scientiae Studia, 9(1), 11-27. https://www.scielo.br/ scielo.php?script=sci_arttext\&pid=S1678-31662011000100002

Serrano, A. (2007). Herculine Barbin llamada Alexina B. Presentado por Michael Foucault. Talasa.

Sinclair, A.H., Berta, P., Palmer, M.S., Hawkins, J., Ross, Griffiths. B.L., Smith, M.J., Foster, J.W., Frischauf, A.-M., Lovell-Badge, R. y Goodfellow, P.N. (1990). A gene from the human sex-determining region encodes a protein with homology to a conserved DNA-binding motif. Nature, 346 (6281) 240244. https://doi.org/10.1038/346240a0

Swain, A. y Lovell-Badge, R. (1997). A molecular approach to sex determination in mammals. Acta Paediatrica (Oslo): Supplemment, 423, 46-49. https://doi. org/10.1111/j.1651-2227.1997.tb18368.x

Valenzuela, N. (2008). Sexual development and the evolution of sex determination. Sexual Development, 2(2), 64-72. https://doi.org/10.1159/000129691

Wilhelm, D., Yang, J. y Thomas, P. (2013). Mammalian sex determination and gonad development. Current Topics in Developmental Biology, 106, 89-121. https://doi.org/10.1016/B978-0-12-416021-7.00003-1 